\title{
Watchful Waiting after Radiochemotherapy in Rectal Cancer: When Is It Feasible?
}

\author{
Cihan Gani ${ }^{a, b} \quad$ Andreas Kirschniak ${ }^{c}$ Daniel Zips ${ }^{a, b}$ \\ ${ }^{a}$ Department of Radiation Oncology, University Hospital Tübingen, Tübingen, Germany; ${ }^{b}$ German Cancer Research \\ Center (DKFZ) Heidelberg and German Consortium for Translational Cancer Research (DKTK), Partner Site Tübingen, \\ Tübingen, Germany; ${ }^{C}$ Department of Surgery, University Hospital Tübingen, Tübingen, Germany
}

\section{Keywords}

Cancer - Complete response - Organ preservation . Radiotherapy $\cdot$ Rectal cancer

\section{Abstract}

A "watch and wait" strategy in rectal cancer is increasingly considered in patients who achieve an excellent response to radiotherapy. While a growing number of studies have shown the feasibility of this strategy in selected patients, the optimal therapeutic regimen to maximize response rates still needs to be established. Furthermore, accurate response prediction and the management of minor residual findings after radiotherapy remain a matter of debate. Finally, concerns regarding the long-term oncological safety of the "watch and wait" approach have been expressed. Therefore, the present review aims to address the open questions in the context of a "watch and wait" strategy and focuses on the diagnosis of a clinical complete response and the ideal management thereafter.

단 2019 S. Karger AG, Base

\section{Introduction}

Organ preservation ("watch and wait") in rectal cancer is an evolving alternative to radical surgery after a clinical complete response (cCR) to neoadjuvant treatment with the promise of improved quality of life and comparable cure rates $[1,2]$. However, with conventional radioche- motherapy protocols the pathological complete response (pCR) rate in patients with locally advanced rectal cancer is only in the range of approximately $10-20 \%$ [3]. Therefore, a major focus in clinical research over the last years was to develop novel and innovative treatment protocols that can increase complete response rates. For this purpose, modifications of concomitant chemotherapy, radiotherapy dose escalation, hyperthermia, or the prolongation of the interval from the end of radiotherapy to surgery have been studied and have shown promising results [3-6]. However, even if response rates can be increased, the accurate prediction of this response is crucial in order to avoid unnecessary surgery in patients who actually have no residual tumor and also nonoperative management in patients who harbor residual tumor cells. Another challenge is the early detection of local recurrences after a cCR is diagnosed, and therefore a "watch and wait" strategy was initiated. In the context of "watch and wait" strategies the term "local regrowth" has been established for this purpose. Our group has previously reviewed the literature regarding these challenges [7]. The present review provides an update, covering the literature that has emerged since, and focuses on the optimal procedure after a cCR is diagnosed.

\section{"Planned" Organ Preservation or "Incidental" cCR?}

The distinction between a "planned" organ preservation strategy and an "incidental" cCR at restaging is crucial.

\section{KARGER}

(c) 2019 S. Karger AG, Basel 
In the early reports of organ preservation in rectal cancer the majority of patients had an indication for "preoperative" radiotherapy due to tumor stage and were treated with standard protocols $[8,9]$. Patients might have been informed about the option of deferring surgery for the first time after restaging before surgery when an "incidental" cCR was seen. Therefore, there was no risk of overtreatment by "preoperative" treatment. Several national and international guidelines now consider a "watch and wait" strategy as an option for patients with an "incidental" cCR - pointing out that the lack of large prospective or randomized series needs to be discussed with the patient (German S3 guideline, ESMO guideline).

A completely different "risk-benefit" analysis has to be conducted when nonoperative management is the predefined treatment goal, and modified radiotherapy protocols to increase the likelihood of a cCR are used or if any radiotherapy is given in stages where surgery could be the only treatment (UICC stage I or "low risk" UICC stage II). For instance, a patient with early distal rectal cancer (stage cT2 cN0 cM0) that would require abdominoperineal resection (APR) has a reasonable chance to achieve a cCR but also the highest risk of overtreatment when radiotherapy is applied with the goal of organ preservation, since an indication for preoperative treatment is not given based on tumor stage $[10,11]$. At the same time the patient in this scenario has the highest potential gain if a cCR is achieved and maintained.

Such a "planned" organ preservation strategy should only be offered in well-designed prospective trials that carefully track long-term oncological outcome and quality of life data.

\section{How to Select Patients for a "Watch and Wait" Strategy? And When Is the Optimal Time Point for Response Assessment?}

Several factors regarding patient- and tumor-related factors need to be considered when selecting patients for a "watch and wait" strategy. We at our departments are very restrictive with discussing a nonoperative strategy in patients who present with a cT4 tumor at baseline, since only a minority of patients in the reported organ preservation trials had cT4 tumors and a higher T-stage appears to be a predictor for local regrowth [12-14]. On the other hand, one could argue that a large cT4 tumor that reaches a cCR has proven a very radiosensitive and favorable biology. However, concerns that a local regrowth could originate from residual vital tumor cell clones in deeper layer of the rectum or adjacent organs overweight in our opinion. Baseline sphincter function is another important factor since the benefit of an organ preservation treatment is very limited for patients who already present with fecal incontinence. Furthermore, patients need to be informed about the need of a regular surveillance if a cCR is achieved and the risk for local regrowth which is approximately $25 \%$ [13].

A major challenge with the implementation of a "watch and wait" strategy is the accurate prediction of a "pathological" complete response. The most commonly used modalities are endoscopy and magnetic resonance imaging (MRI). In their report of 21 patients managed nonoperatively, Maas et al. [9] applied very strict criteria for the definition of a cCR, requiring the absence of any residual tumor on MRI and only permitted a small residual ulcer or scar on endoscopy. With these criteria only 1 of 21 patients developed local regrowth during surveillance after surgery was omitted. However, out of 20 patients who underwent surgery and actually had a pCR, 15 were judged to have a residual tumor on MRI. Endoscopy alone has a high positive predictive value, which means that a white scar or small residual ulcer on endoscopy predicting a "true pCR" is present in $90 \%$ of cases. The Achilles heel however is the sensitivity. In a study comparing the sensitivity of endoscopy and MRI to detect a true pCR, endoscopy outperformed MRI (53 vs. 35\%) [15]. Over the last years, the "near clinical complete response" has emerged as a third category in response assessment besides "poor response/residual tumor" and "clinical complete response." The underlying rationale is the observation that a significant proportion of residual findings such as "small residual flat ulcer, or irregular wall thickening" in endoscopy seen at a first response evaluation 8-10 weeks after the end of radiochemotherapy do convert into a cCR with only a white scar with telangiectasia after another 6-12 weeks [16]. In the ongoing CAO/ ARO/AIO-16 trial (NCT03561142) we have implemented the concept of a "near clinical complete response" with a repeated evaluation after 3 months and base the response definition regarding the primary tumor solely on findings on endoscopy, reserving MRIs for the assessment of mesorectal lymph nodes. Figure 1 provides examples of a cCR, a near CCR, and a poor response on restaging after radiochemotherapy.

\section{What Is the Optimal Surveillance Strategy?}

If the decision for the omission of surgery has been made after a cCR was diagnosed, regular surveillance studies have to be conducted. Approximately $25 \%$ of patients will develop local regrowth and the majority of these can be salvaged given a timely detection $[10,13,17]$. Endoscopy is the most relevant modality for surveillance since in most studies $>95 \%$ of local regrowths were seen in the rectal wall [18]. Nodal failures in the mesorectum are less frequent or even absent in some trials but have 

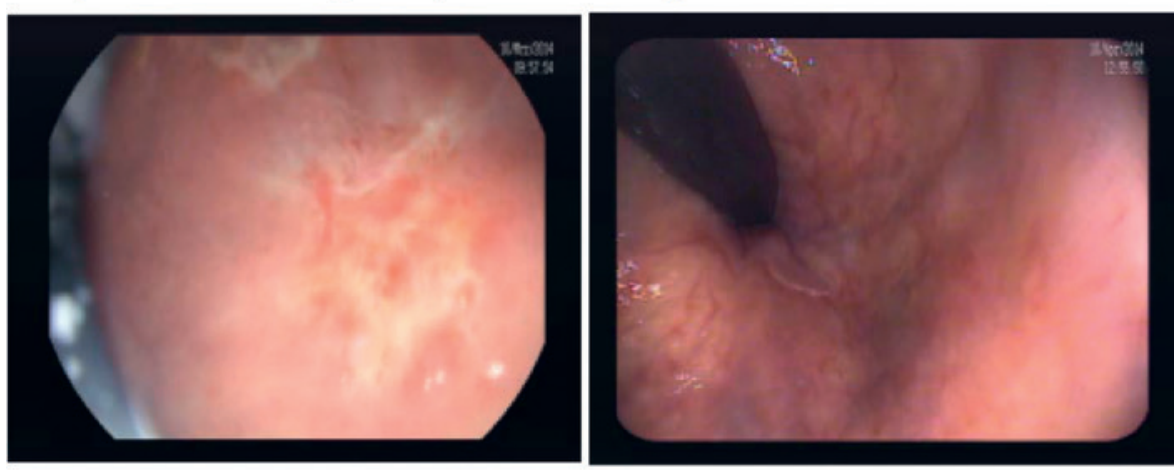

Examples for a near clinical complete response on endoscopy::
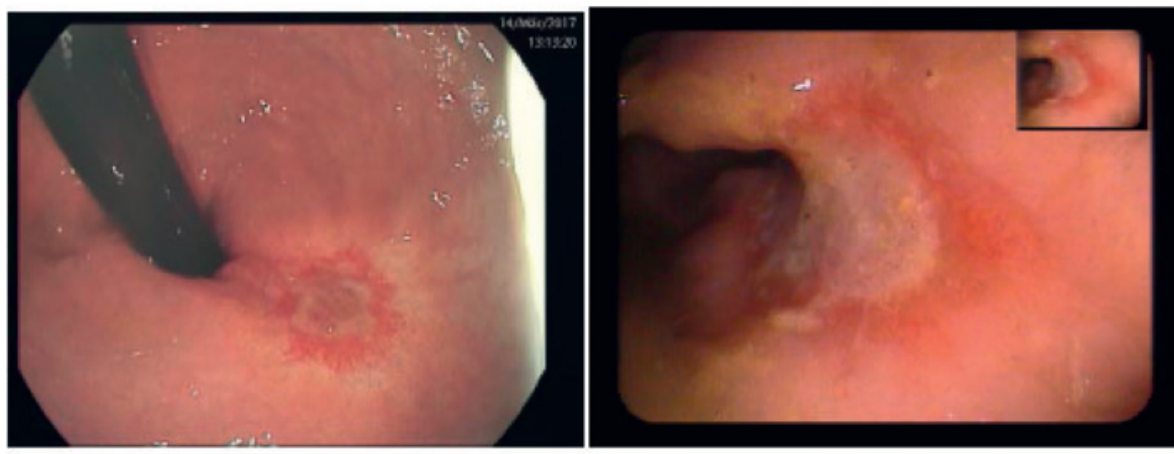

Examples for poor response or residual tumor on endoscopy::

Fig. 1. Examples of a clinical complete response, a near clinical complete response, and a poor response on restaging after radiochemotherapy.

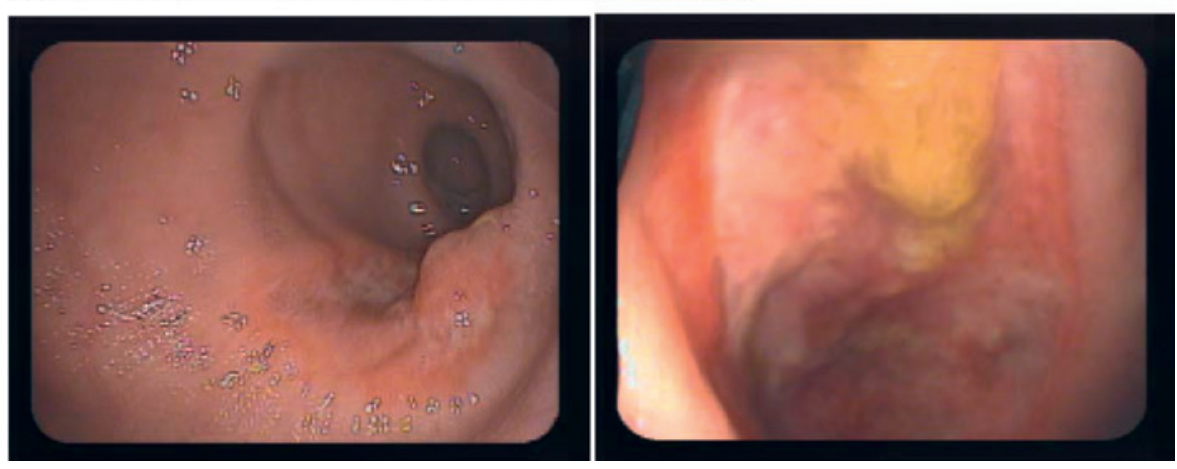

also been reported at higher incidences as well [17]. The majority of local regrowths develop within the first 2 years of surveillance; however, follow-up with risk-adapted intervals should be performed over 5 years. We therefore recommend 3 monthly endoscopies and MRIs in the first 2 years, 4 -monthly in the third year, and 6-monthly in years 4 and 5 .

\section{Is Limited Surgery an Option?}

It is intuitive to consider a "limited" surgical approach with local excision for patients who have responded well to radiotherapy but still show a residual finding of unclear dignity [19]. The advantage is the removal of the residual finding and the histological confirmation in a single procedure that can still permit sphincter preservation in distal tumors. On the other hand, completion surgery with low anterior resection or APR is generally recommended when pathological evaluation of the locally excised sample results in a ypT2-3 or ypN+or $\mathrm{R} 1$ resection since there is a considerable risk of local recurrence from lymph nodes or the involved margin. In a prospective trial Bujko et al. [20] treated 89 patients with cT1, cT2, or "borderline" cT2/3 cN0 with either short-course radiotherapy $(5 \times 5$ Gy and 4-Gy boost) or radiochemotherapy with $55.8 \mathrm{~Gy}$ and 5-FU and leucovorin. At 6-8 weeks after radiotherapy a local excision was performed, which was the definitive treatment in case of a pCR or a ypT1 stage without risk factors (positive margin, fragmented tumor, G3, 
venous, perineural, or lymphatic vessel affection). In all other cases completion surgery with APR or total mesorectal excision (TME) was recommended. A pCR or ypT1 stage with no indication for completion surgery was seen in $63 / 89$ patients $(70.8 \%)$. Of the remaining 26 patients who required completion surgery, 18 patients either refused completion or were deemed unfit for the procedure. The local failure rate in this subgroup was 8/18 (44.4\%). In the subgroup of patients who did not require immediate "completion" TME, the local failure rate was 5/63 (7.9\%). Since the absolute total number of local failures was low, no firm conclusions from subgroup analyses could be drawn [20].

The recently reported multicentric GRECCAR-2 trial randomized 145 patients with T2 or T3 tumors (maximum size $4 \mathrm{~cm}$ ) in the lower rectum that had shown a good response after radiochemotherapy and had shrunken to $2 \mathrm{~cm}$ or less to either local excision or TME. A composite endpoint of death, all recurrences, morbidity, and side effects after 2 years was defined and should prove the superiority of local excision over TME. Completion TME was recommended in case of yT2 or ypT3 tumors after local excision. The trial failed to show the superiority of local excision over TME, which was mainly due to the 26 of 74 patients in the local excision arm who required completion TME. In a post hoc analysis major morbidity and side effects occurred in $78 \%$ of patients who underwent completion TME after local excision compared with 38\% in the TME arm [11]. While the trial confirmed favorable failure and organ preservation rates reported before in the CARTS and ACOSOG Z6041, GRECCAR-2 trial underlines the importance of accurate restaging after radiochemotherapy in order to prevent major morbidity due to completion surgery $[21,22]$.

\section{Could We Harm Our Patients?}

One of the concerns expressed by both colleagues and patients is that an initially curable localized disease might become incurable due to distant spread originating from local regrowth. Another concern is the need for a more radical surgical approach such as extralevatoric APR or even pelvic exenteration for local regrowth while initially a sphincter-preserving TME would have been sufficient.

Martens et al. [17] provide a very detailed failure pattern of 100 patients who had been treated nonoperatively after a complete response to radiotherapy. Of these 100 patients, 15 developed local regrowth after a median follow-up of more than 3 years. Twelve of these 15 local regrowths were isolated without distant metastases and easily salvaged surgically. Importantly, the required surgical procedure for salvage was no more radical than the one that would have been chosen immediately after radiotherapy. One patient who had undergone transanal endoscopic microsurgery after radiochemotherapy required pelvic exenteration for an isolated local regrowth that had developed 13 months after radiotherapy. This single patient was clearly disadvantaged by the "watch and wait" strategy. However, it should be noted that transanal endoscopic microsurgery had shown a yp T2 stage, and generally an immediate completion surgery is recommended due to the high risk of disease progression in these patients [17]. Regarding distant failures after nonoperative management in a meta-analysis of 23 studies and $867 \mathrm{pa}-$ tients, Dossa et al. [23] estimate that per 1,000 patients treated nonoperatively, 1 patient per 1,000 will not undergo salvage surgery due to concomitant systemic disease. However, among patients who develop local regrowths the incidence of distant metastases either at the time of diagnosis of local regrowth or during further follow-up is clearly higher and was 13\% ( 2 of 15 patients with local regrowths) in the previously mentioned Dutch study, but as high as $36 \%$ ( 8 of 22 patients with local regrowths) in a series from Memorial Sloan Kettering Cancer Center [18]. The crucial question here is whether these metastases were already present at a microscopic degree before preoperative treatment was initiated or whether they developed from a clone that survived radiotherapy and led to a local regrowth reflecting its biological aggressiveness. In the absence of studies that randomized patients with a cCR to immediate surgery or nonoperative management this question cannot be answered and has to be discussed in a shared decision making with the patient.

\section{Conclusion and Perspective}

With novel innovative treatment protocols an increasing number of rectal cancer patients will qualify for nonoperative management. Endoscopy is the preferred method for the definition of a cCR. A "near CCR" with small residual findings on an initial evaluation after radiotherapy has a considerable chance of transforming into a cCR on reevaluation after 3 more months. Local excision can lead to sphincter preservation in carefully selected patients but bears the risk of increased morbidity and side effects when completion TME is indicated. Long-term oncological outcomes after a cCR and deferred strategy are favorable; however, the lack of randomized data regarding local and distant failure rates should be discussed with patients before a "watch and wait" strategy is established. Future research will have to optimize the accuracy of response prediction. Furthermore, more data regarding quality of life and bowel function after organ preservation are necessary. 


\section{Disclosure Statement}

The Department of Radiation Oncology Tübingen receives financial and technical support from Elekta AB (Stockholm, Sweden) under a research agreement and financial support for educa- tional events from Dr. Sennewald GmbH (Munich, Germany). The surgical training center Tübingen received support from Karl Storz Endoskopie GmbH (Tuttlingen), Takeda, Medtronic, Ethicon, and Erbe Elektromedizin (Tübingen). A.K. Received honoraria for talks from Falk, Takeda, and Jansen.

\section{References}

1 Hupkens BJ, Martens MH, Stoot JH, Berbee M, Melenhorst J, Beets-Tan RG, et al. Quality of Life in Rectal Cancer Patients After Chemoradiation: Watch-and-Wait Policy Versus Standard Resection - A Matched-Controlled Study. Dis Colon Rectum. 2017 Oct;60(10): 1032-40.

2 Smith JD, Ruby JA, Goodman KA, Saltz LB, Guillem JG, Weiser MR, et al. Nonoperative management of rectal cancer with complete clinical response after neoadjuvant therapy. Ann Surg. 2012 Dec;256(6):965-72.

3 Rödel C, Liersch T, Becker H, Fietkau R, Hohenberger W, Hothorn T, et al.; German Rectal Cancer Study Group. Preoperative chemoradiotherapy and postoperative chemotherapy with fluorouracil and oxaliplatin versus fluorouracil alone in locally advanced rectal cancer: initial results of the German CAO/ ARO/AIO-04 randomised phase 3 trial. Lancet Oncol. 2012 Jul;13(7):679-87.

4 Gani C, Schroeder C, Heinrich V, Spillner P, Lamprecht $U$, Berger B, et al. Long-term local control and survival after preoperative radiochemotherapy in combination with deep regional hyperthermia in locally advanced rectal cancer. Int J Hyperthermia. 2016;32(2): 187-92.

5 Schroeder C, Gani C, Lamprecht U, von Weyhern $\mathrm{CH}$, Weinmann $\mathrm{M}$, Bamberg $\mathrm{M}$, et al. Pathological complete response and sphincter-sparing surgery after neoadjuvant radiochemotherapy with regional hyperthermia for locally advanced rectal cancer compared with radiochemotherapy alone. Int J Hyperthermia. 2012;28(8):707-14.

6 Garcia-Aguilar J, Chow OS, Smith DD, Marcet JE, Cataldo PA, Varma MG, et al.; Timing of Rectal Cancer Response to Chemoradiation Consortium. Effect of adding mFOLFOX6 after neoadjuvant chemoradiation in locally advanced rectal cancer: a multicentre, phase 2 trial. Lancet Oncol. 2015 Aug;16(8):957-66.

7 Gani C, Bonomo P, Zwirner K, Schroeder C, Menegakis A, Rödel C, et al. Organ preservation in rectal cancer - Challenges and future strategies. Clin Transl Radiat Oncol. 2017 Mar;3:9-15.

8 Habr-Gama A, Perez RO, Nadalin W, Sabbaga J, Ribeiro U Jr, Silva e Sousa AH Jr, et al. Operative versus nonoperative treatment for stage 0 distal rectal cancer following chemoradiation therapy: long-term results. Ann Surg. 2004 Oct;240(4):711-7; discussion 717-8.

9 Maas M, Beets-Tan RG, Lambregts DM, Lammering G, Nelemans PJ, Engelen SM, et al. Wait-and-see policy for clinical complete responders after chemoradiation for rectal cancer. J Clin Oncol. 2011 Dec;29(35):4633-40.

10 Appelt AL, Pløen J, Harling H, Jensen FS, Jensen LH, Jørgensen JC, et al. High-dose chemoradiotherapy and watchful waiting for distal rectal cancer: a prospective observational study. Lancet Oncol. 2015 Aug;16(8):919-27.

11 Rullier E, Rouanet P, Tuech JJ, Valverde A, Lelong B, Rivoire M, et al. Organ preservation for rectal cancer (GRECCAR 2): a prospective, randomised, open-label, multicentre, phase 3 trial. Lancet. 2017 Jul;390(10093): 469-79.

12 Renehan AG, Malcomson L, Emsley R, Gollins S, Maw A, Myint AS, et al. Watchand-wait approach versus surgical resection after chemoradiotherapy for patients with rectal cancer (the OnCoRe project): a propensity-score matched cohort analysis. Lancet Oncol. 2016 Feb.;17(2):174-83.

13 van der Valk MJ, Hilling DE, Bastiaannet E, Meershoek-Klein Kranenbarg E, Beets GL, Figueiredo NL, et al.; IWWD Consortium. Long-term outcomes of clinical complete responders after neoadjuvant treatment for rectal cancer in the International Watch \& Wait Database (IWWD): an international multicentre registry study. Lancet. 2018 Jun; 391(10139):2537-45.

14 Chadi SA, Malcomson L, Ensor J, Riley RD, Vaccaro CA, Rossi GL, et al. Factors affecting local regrowth after watch and wait for patients with a clinical complete response following chemoradiotherapy in rectal cancer (InterCoRe consortium): an individual participant data meta-analysis. Lancet Gastroenterol Hepatol. 2018 Dec;3(12):825-36.

15 Maas M, Lambregts DM, Nelemans PJ, Heijnen LA, Martens MH, Leijtens JW, et al. Assessment of Clinical Complete Response After Chemoradiation for Rectal Cancer with Digital Rectal Examination, Endoscopy, and MRI: Selection for Organ-Saving Treatment. Ann Surg Oncol. 2015 Nov;22(12):3873-80.

16 Hupkens BJ, Maas M, Martens MH, van der Sande ME, Lambregts DM, Breukink SO, et al. Organ Preservation in Rectal Cancer After Chemoradiation: Should We Extend the Observation Period in Patients with a Clinical Near-Complete Response? Ann Surg Oncol. 2018 Jan;25(1):197-203.

17 Martens MH, Maas M, Heijnen LA, Lambregts DM, Leijtens JW, Stassen LP, et al. Long-term Outcome of an Organ Preservation Program after Neoadjuvant Treatment for Rectal Cancer. J Natl Cancer Inst. 2016 Aug;108(12). pii: djw171.

18 Smith JJ, Strombom P, Chow OS, Roxburgh CS, Lynn P, Eaton A, et al. Assessment of a Watch-and-Wait Strategy for Rectal Cancer in Patients with a Complete Response after Neoadjuvant Therapy. JAMA Oncol. 2019 Jan:e185896.

19 van der Sande ME, Beets GL, Hupkens BJ, Breukink SO, Melenhorst J, Bakers FC, et al. Response assessment after (chemo)radiotherapy for rectal cancer: why are we missing complete responses with MRI and endoscopy?EurJSurgOncol.2018Nov;S0748-7983(18) 32017-1.

20 Bujko K, Richter P, Smith FM, Polkowski W Szczepkowski M, Rutkowski A, et al. Preoperative radiotherapy and local excision of rectal cancer with immediate radical re-operation for poor responders: a prospective multicentre study. Radiother Oncol. 2013 Feb; 106(2):198-205.

21 Verseveld M, de Graaf EJ, Verhoef C, van Meerten E, Punt CJ, de Hingh $\mathrm{IH}$, et al. Chemoradiation therapy for rectal cancer in the distal rectum followed by organ-sparing transanal endoscopic microsurgery (CARTS study). Br J Surg. 2015 Jun;102(7):853-60.

22 Garcia-Aguilar J, Renfro LA, Chow OS, Shi Q, Carrero XW, Lynn PB, et al. Organ preservation for clinical T2N0 distal rectal cancer using neoadjuvant chemoradiotherapy and local excision (ACOSOG Z6041): results of an open-label, single-arm, multi-institutional, phase 2 trial. Lancet Oncol. 2015 Nov;16(15): 1537-46.

23 Dossa F, Chesney TR, Acuna SA, Baxter NN. A watch-and-wait approach for locally advanced rectal cancer after a clinical complete response following neoadjuvant chemoradiation: a systematic review and meta-analysis. Lancet Gastroenterol Hepatol. 2017 Jul;2(7): 501-13. 